\title{
Supercritical Fluid Chromatographic Separation of Dimethylpolysiloxane Polymer
}

\author{
Dongjin Pyo* and Changhyun Lim

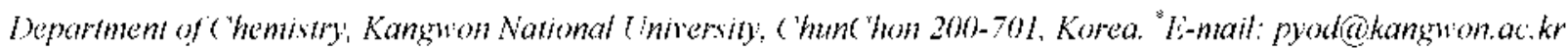 \\ Recerved Nowenber t, 2004
}

Key Words : Supercritical fluid chromatography, Dimethylpolysiloxane

The use of compressed (dense) gases and supercritical fluids as chromatographic mobile phases in conjunction with liquid chromatographic (LC)-type packed columns was first reported by Klesper of al. in 1962.' During its relatively short history, supercritical fluid chromatograply (SFC) has become an attractive alternative to $\mathrm{GC}$ and $\mathrm{LC}$ in certain industrially important applications. SFC gives the advantage of high efficiency and allows the analysis of nonvolatile or themally labile mixtures.

The density of the mobile phase in SFC is about 200-500 times that in gas chromatography. The effect of shorter internolecular distances and the resulting increase in molecular interactions is an enhanced solubilizing capability of the solvent towards various solutes. Compounds with much higher molecular weights than gas chromatography nomally allows can therefore be chromatographed. However, the most commonly used mobile phases in SFC are all relatively non-polar fluids. Carbon dioxide, the most widely used fluid, is no more polar than hexane ${ }^{2}$ even at high densities. Solute polarity should be between that of the stationary plase and the mobile phase in order to have a well behaved separation. Few real samples contain only nonpolar solutes, so a major objective of research in $\mathrm{SFC}$ has been directed toward increasing the range of solute polarity that can be handled by the technique. To bring the SFC technique into routine use, mobile phases that are more polar than the commonly used carbon dioxide are necessary.

The solvent strength of supercitical $\mathrm{CO}_{2}$, even at high density, is not sufficient for the elution of polar solutes. Polar mobile phase such as $\mathrm{VH}_{i}{ }^{4}$ exhibit useful properties, but a more practical way to extend the range of compounds separable by $\mathrm{SFC}$ is to use a mixed mobile phase. The solubility of the solute in the supercritical plase can be influenced considerably by adding modifiers to the mobile phase. The use of modifiers has been reported by Jentoft and Gouw'i and by . Vovotny of al. ${ }^{+}$The latter group showed that adding $0.1 \%$ 2-propanol to pentane as the mobile phase decreases the observed partition coefficient $(k)$ values for many polynuclear aromatic hydrocarbons by $20-35 \%$. Thus, the addition of modifiers (generally organic solvents) to a supercritical mobile phase changes the polarity of the mobile phase and also leads to deactivation of the column packing material. In capillary SFC, most separations are made with pure $\mathrm{CO}_{2}$ because of its compatibility with an flame ionization detection (FID), except for formic acid and water. the addition of any common modifier precludes the use of FID. ${ }^{5}$ Modifiers are essential in packed-column SFC for the elution of polar compounds" and are extensively used.

Several workers ${ }^{t+k}$ have reported the influence of modifiers on peak shape, selectivity and retention time in capillary and packed-column SFC. A simple and effective way to add modifiers to a supercitical fluid mobile phase is to use a saturator column? ${ }^{\text {?.1!! }}$ which is usually a silica column saturated with polar alcoliols.

In this work, water was used as a polar modifier and a $\mu$ porasil column as a saturator column. The $\mu$-porasil column was inserted between the pump outlet and the injection valve. During the passage of the supercritical fluid mobile phase through the silica column, a polar modifier (water) can be dissolved in the pressurized supercritical tluid. Dimethylpolysiloxane polymer has been known as more polar polymer than polystyrene polymer. ${ }^{12}$ Dimethylpolysiloxane polymer has never been separated using water modified mobile phase. In this paper, using a $\mu$-porasil column as a saturator column, excellent supercritical fluid chromatogams of dimethylpolysiloxane oligomers were obtained.

\section{Experiments}

A HP (Hewlett Packard, Palo Alto. CA) Model 5890 gas chromatograph was reconstructed in the laboratory as a supercritical fluid clironatograph. The supercritical column used was a $100 \mathrm{~mm} \times 2 \mathrm{~mm}$ i.d. packed column (.Vucleosil diol). This system was equipped with a C14W loop injector

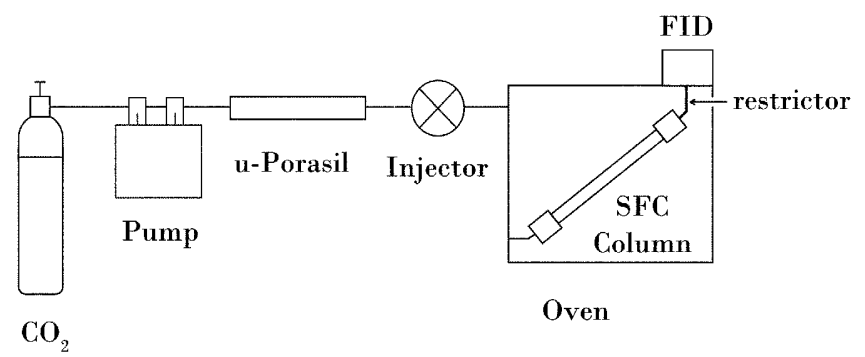

Figure 1. Schematic diagran of supercritical flud chromatography system used for the separation of dimethylpolysiloxane polymer. 
(Valco) and a flame ionization detector. SFC-grade carbon dioxide (Scott Specialty Gases) was used as a basic mobile phase. Supercritical expenimental conditions are the followings; the temperature was maintained at $150^{\circ} \mathrm{C}$, pressure

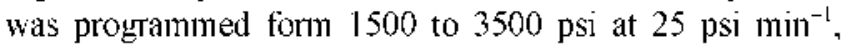
detector at $300{ }^{\circ} \mathrm{C}$ and $5 \mathrm{~mL} \mathrm{~min}{ }^{-1}$ restrictor flow-rate at $1500 \mathrm{psi}$. For the addition of modifiers 10 supercritical $\mathrm{CO}_{2}$, at $\mu$-Porasil column $(250 \mathrm{~mm} \times 4.6 \mathrm{~mm}$ i.d.) which is manufactured for nomal-phase HPLC by Waters was used. Its functional group is a silanol ( $\mathrm{SiOH}$ ) group. The $\mu$-Porasil column was saturated with modifiers using a Model 600 syringe pump (Lee Scientific) and placed between the punp and injector. Figure I shows the schemalic diagram of supercritical fliud chromatography system used for the separation of dimethylpolysiloxane polymer.

\section{Results and Discussions}

When modifiers are used with supercritical $\mathrm{CO}_{2}$ in order to chromatograph more polar substances, the binary mixture of eluents can contaminate the instrunnent. Especially when water or formic acid is used as a modifier, the modifier remaining in a pump may cause corrosion of the pump, and when methanol is used as a modifier. methanol remaining in the pump can be eluted slowly during the next run. This may affect the time required to achieve chemical equilibrium for the subseguent separations, and also many modifiers can cvaporate and contaminate the air in the laboratory. A good way to overcome these problems is to use a saturator column ${ }^{9.17 .11}$ to add polar modifiers to supercritical $\mathrm{CO}_{2}$. For the experiment, water was used as a modifier and a $\mu$-Porasil column was used as a saturator column with system desing similar to that described by Engelhardt $e t a f{ }^{\text {" }}$ With this desing, a polar modilier (water) can be added to pressurized $\mathrm{CO}_{2}$ after the pump, and thus no modifier remains in the pump. Supercritical $\mathrm{CO}_{2}$ is delivered from the pump to the "t-Porasil column which is salurated with water. When

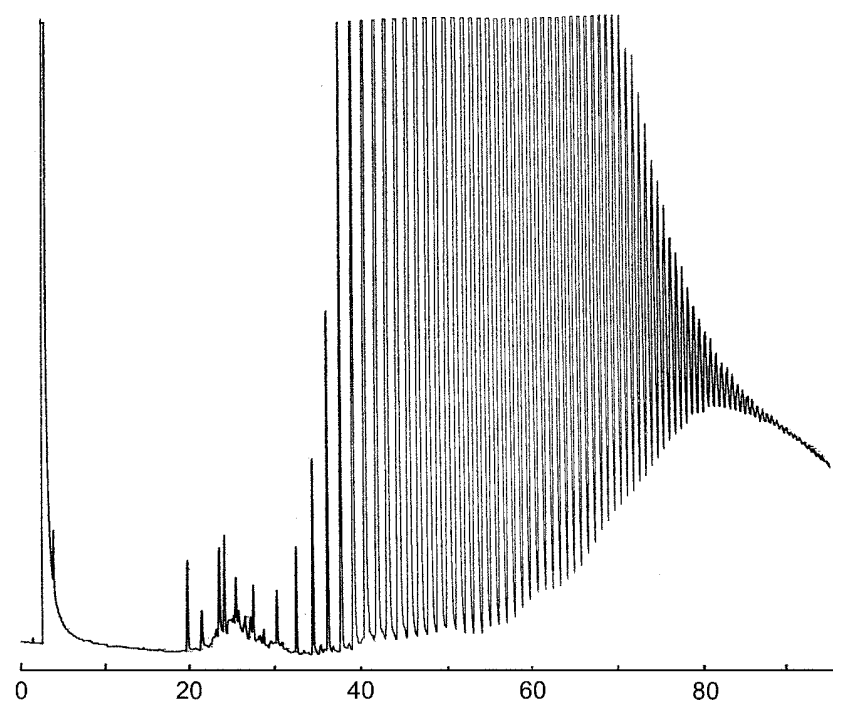

Figure 2. Chromatogram of oligomers of dimethylpolysiloxane (No. 1) using a $\mu$-Porasil column. supercritical $\mathrm{CO}_{2}$ passes through the $\mu$-Porasil column. water molecules held on the $\mathrm{OH}$ groups of the $\mu$-Porasil by bydrogen bonding can dissolve in the pressurized supercritical fluid. Thus non-polar supererilical $\mathrm{CO}_{2}$ can have the characteristics of a polar mobile phase because it can absorb a polar solvent, i.e. water. Therefore, after passing through the $\mu$-Porasil column, supercritical $\mathrm{CO}_{2}$ is changed into a new mobile phase with different polarity, and it is possible to separate polar samples using this modified mobile phase. Dimethylpolysiloxane oligomers were analysed with this modified mobile phase (supercritical carbon dioxide-water phase) using a $\mu$-Porasil column as saturator column. The chromatograms are shown in Figure 2 and Figure 3. Excellent separations were obtained. These kinds of separations are almost impossible with other chromatographic techniques. Figure 4 and Figure 5 show the separation of

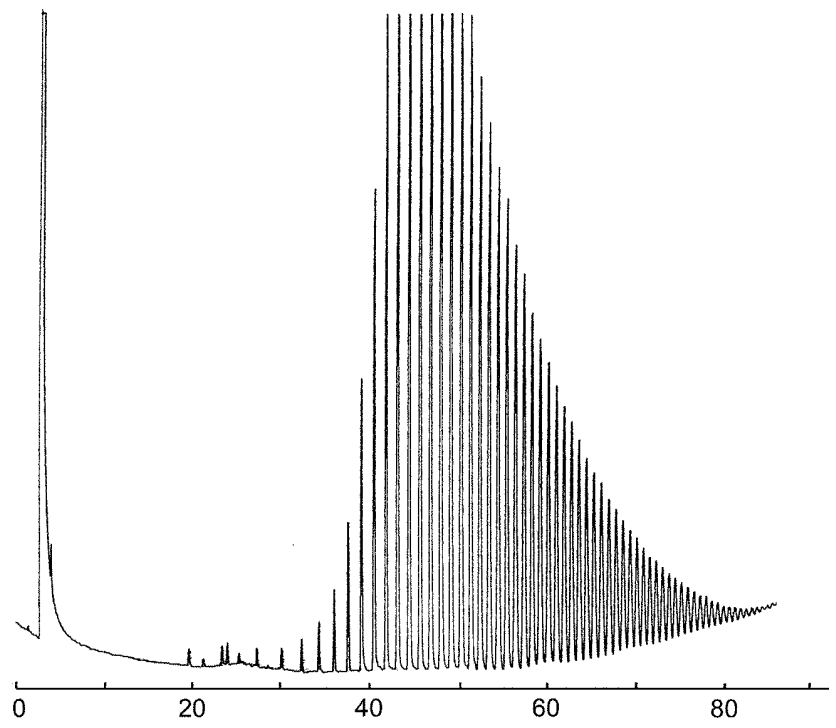

Figure 3. Chromatogram of oligomers of dimethylpolysiloxane (No. 2) using a $\mu$-Porasil column.

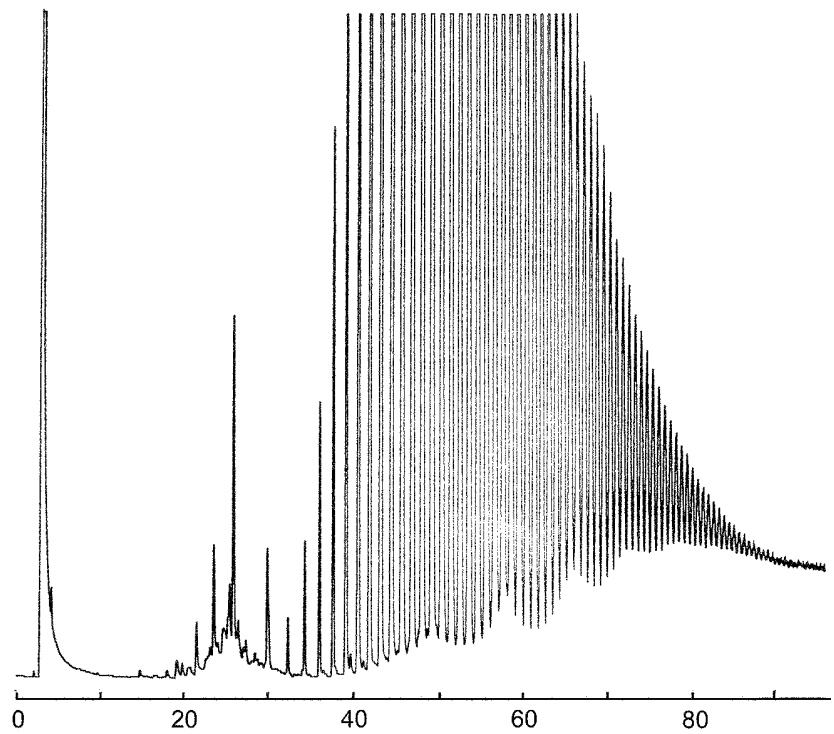

Figure 4. Chromatogram of oligomers of dimethylpolysiloxane (No. 1) without using a $\mu$-Porasil column. 


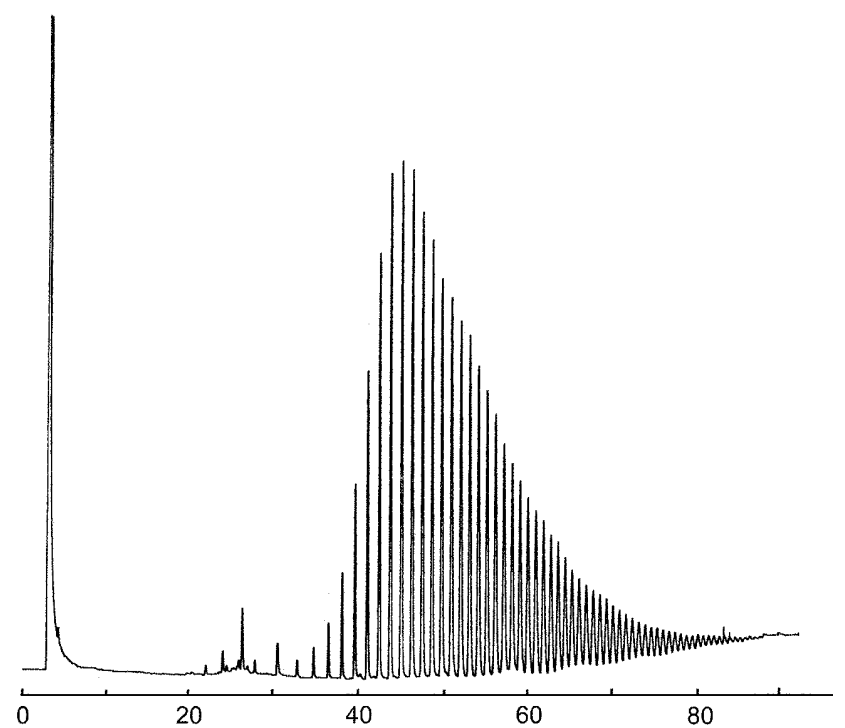

Figure 5 Chromatogram of oligomers of dimethylpolysiloxane (No. 2) without using a $\mu$-Porasil column.

dimelhylpolysiloxane oligomers with pure carbon dioxide as the mobile phase. As expected, when a $\mu$-Porasil column was used. more peaks were separated. The numbers of peaks scparated are the followings; 75 peaks in Figure 2, 65 peaks in Figure 3, 80 peaks in Figure 4,69 peaks in Figure 5. These results agree well with those reported by Hirata. ${ }^{12}$ In Hirata's experiment, when $10 \%$ ethanol was added on nonpolar mobile phase, more oligomers were eluted. This may be due to the polarity of dimethylpolysiloxane polymer.

\section{Conclusion}

The excellent supercritical fluid chromatograms were obtained for the separations of dimethylpolysiloxane polymers. When small amount of water was added on supercritical carbon dioxide fluid, more oligomer peaks were observed. After passing litrough the $\mu$-Porasil column, supercritical carbon dioxide is changed into a new mobile phase with different polarity, and it was possible to elute more oligomers using this modified mobile phase

Acknowledgment. The authors gratefully acknowledge the financial support from the Korea Ministry of Commerec, Industry \& Energy and the Korea Energy Management Corporation to supercritical fluid project. This work was also supporled by a grant from Basic Science Rescarch Institute at Kangwon National University.

\section{References}

J. Klesper, E.; Corwin, A. H.: Turner, D. A. J. (O)g. (hem. 1962, 27, 700.

2. Yonker, C. R.; Frye, S. L.; Lalkwarf, D. R., Smith, R. D. f. Thws (them. 1986, 90, 302

3. Jentoft, R. E.; Gouw T. H. . ( hrmarogr Sci. 1970, \&, 138

4. Novotny, M.: Bertsch. W; Zlatkis A. I. (hromatogr: 1971, 6/, 17

5. Wright, B. W.: Smith, R. D. J Chromatogr: 1986, 355, 367.

6. Schmidt, S ; Blomberg, L. G; Campbell, E. R. Chromatographia $1988,25,775$

7. Blilie, A. L., Greibrohk, T Anal. ('hem. 1985, 57, 2239

8. Yonker, C. R.: Smith, R. D. . ( Wromatogr: 1986, 36), 25

9. Engelhardt, II: Gross, A; Metens, R: Petersen, M. J. (hromatogr: 1989, $+77,169$.

10. Schwartz. H. E.; Barthel, P. J.: Moring, S. E.; Yates, T. L.: Lauer, H. H. Tresenim. ' Anal ( Them. 1988, 330, 204

11. Karger. B. L.; Castells, R. C.: Sewell, P. A; Hartkopf, A. J. /hts. ( hem. 1971, 75, 3870 .

12. Hirata, Y. /, ('hematog): $1984,315,39$ 